\title{
Immunological effect of ten-year c-ART in treatment-naive and pre-treated HIV-1 patients in Bulgaria
}

\author{
Ivaylo Elenkov ${ }^{1 \dagger}$, Maria Nikolova ${ }^{2^{*}}$, Ivanka Radeva ${ }^{1}$, Margarita Yankova ${ }^{1}$, Nina Yancheva ${ }^{1}$ \\ From 17th International Symposium on HIV and Emerging Infectious Diseases (ISHEID) \\ Marseille, France. 23-25 May 2012
}

\section{Introduction}

Highly effective combination anti-retroviral therapy (cART) has been applied in Bulgaria since 1999. The aim of the present study was to compare retrospectively the longterm immunological effect of c-ART in treatment-naïve and pre-treated HIV-1+ patients.

\section{Patients and methods}

The study included HIV-1+ patients $(\mathrm{n}=56)$ that have started c-ART between March 1999 and December 2001, have been on continuous treatment, with good adherence, death being the only reason for ART stop. Of them, 27 had a history of irregular pre-treatment with AZT or AZT/LMV for an average of 4.6 yrs (Group A), and 29 were ART-naïve (Group B). CD4 absolute counts (AC) were determined by single-platform flow cytometry (BD Biosciences). Viral load (VL) was measured by RT-PCR (Roche). Comparisons were performed by unpaired $\mathrm{t}$-test (SPSS 17.0).

\section{Results}

The demographic characteristics of groups A and B did not differ significantly: mean age (yrs): 34 vs. 35 ; male to female ratio: 9 vs. 7, respectively. Baseline CD4 AC (cells/ $\mathrm{ml})$ and VL (log HIV RNA copies/ml) were comparable: mean 124 vs. 119 , and 5.1 vs. 4.6 , respectively, ( $p>0.05$ for both comparisons). In the long term, suppression of viral replication was observed in both groups: mean VL at 5 yrs 3.7 vs. 3.1 for groups A and B, respectively, $(\mathrm{p}>0.05)$. However, treatment-naïve patients (group B) had a better immune recovery than group A, and the difference became significant in the long term: mean CD4 AC 177 vs. 252 after 6 months of c-ART, (p>0.05), 391 vs. 240 at 2 yrs (p

\section{Conclusion}

Similarly to other studies, (SHM Monitoring report, 2009), a more complete and lasting long-term immunologic response to c-ART was observed in treatment - naïve patients. According to us, a previous sub-optimal and irregularly applied ART regimen, may promote the selection of gradually outgrowing drug-resistant viral strains, compromising therapeutic efficacy in the long run.

\section{Author details}

${ }^{1}$ Specialized Hospital for Active Treatment of Infectious and Parasitic Diseases, Sofia, Bulgaria. ${ }^{2}$ Clinical Immunology at National Center of Infectious and Parasitic Diseases, Sofia, Bulgaria.

Published: 25 May 2012

doi:10.1186/1742-4690-9-S1-P12

Cite this article as: Elenkov et al:: Immunological effect of ten-year c ART in treatment-naive and pre-treated HIV-1 patients in Bulgaria. Retrovirology 2012 9(Suppl 1):P12.

\footnotetext{
* Correspondence: mstoimenova11@gmail.com

† Contributed equally

${ }^{2}$ Clinical Immunology at National Center of Infectious and Parasitic Diseases, Sofia, Bulgaria

Full list of author information is available at the end of the article
}

(c) 2012 Elenkov et al; licensee BioMed Central Ltd. This is an Open Access article distributed under the terms of the Creative Commons 